Supporting Information

\title{
Light-activated hypoxia-sensitive covalent organic framework for tandem-responsive drug delivery
}

Lei Ge, Chenyang Qiao, Yikai Tang, Xiaoke Zhang, Xiqun Jiang*,

Department of Polymer Science \& Engineering, College of Chemistry \& Chemical

Engineering, Nanjing University, Nanjing 210023, China

*Corresponding Author Email: jiangx@nju.edu.cn 
Materials: 4,4'-Azodianiline (AD), benzidine (BD), 1, 3, 5-triformyl-2, 4, 6trihydroxybenzene (TP), chlorine e6 (Ce6), and tirapazamine (TPZ) were obtained from J\&K Chemical Ltd. Amino-poly ethylene glycol-methoxy $\left(\mathrm{mPEG}_{\mathrm{NH}}, M w=5000\right)$, trifluoroacetic acid (TFA), sodium hydrosulfite, 2',7'-dichlorodihydrofluorescein diacetate (DCFH-DA), the reduced form of nicotinamide adenine dinucleotide phosphate (NADPH), orange II and solvents were purchased from Sigma-Aldrich Chemical Co. All the commercially available reagents were used as received without any further purification. Image-i $\mathrm{T}^{\mathrm{TM}}$ Hypoxia Reagent and other cell culture products were purchased from Thermo Fisher Scientific unless otherwise stated.

\section{Preparation of TA-COF}

In the first step, in a typical synthesis ${ }^{1}$, the $4,4^{\prime}$-azodianiline (AD) $(0.45 \mathrm{mmol})$ was added into $100 \mathrm{~mL}$ dichloromethane at room temperature, which resulted in a transparent homogeneous solution. Next, trifluoroacetic acid $(10 \mu \mathrm{L})$ was added directly to the solution as the catalyst for Schiff base reaction, turning the color of the solution from dark yellow to wine red. Finally, a solution of $0.30 \mathrm{mmol} 1,3$, 5-triformyl2, 4, 6-trihydroxybenzene (TP) in $100 \mathrm{~mL}$ ethanol was added slowly with the stirring at room temperature. After stirring for $6 \mathrm{~h}$, the suspension was centrifuged at 10000 rpm for $15 \mathrm{~min}$ and the red residue was collected. Then the residue was thoroughly washed with ethanol and dimethylacetamide three times, respectively, followed by dry acetone and isolated as powder after drying in an oven at $120^{\circ} \mathrm{C}$. TB-COF was prepared by $\mathrm{TP}$ and $\mathrm{BD}$ through a similar procedure.

\section{Preparation of TA-COF-P@CT}


$5 \mathrm{mg}$ TA-COF was dispersed in $5 \mathrm{~mL}$ dichloromethane and then the dispersion was drop-wisely added to the dichloromethane solution of $\mathrm{mPEG}-\mathrm{NH}_{2}(M w=5000,1 \mathrm{mg}$ $\mathrm{mL}^{-1}, 20 \mathrm{~mL}$ ) under stirring. After stirring overnight, the precipitated red solids were collected by centrifugation. After drying in air, the solids were dissolved in $20 \mathrm{~mL}$ DI water. After that, Ce6 and TPZ in DMSO $\left(2 \mathrm{mg} \mathrm{mL}^{-1}, 500 \mu \mathrm{L}\right.$, separately) was added and further stirred for $12 \mathrm{~h}$. The resulting solution was centrifuged at $5000 \mathrm{rpm}$ for 5 min to remove the bulk particles, and the collected supernatant was further dialyzed (molecular weight cut off (MWCO) 8000 - 14000) against a mixed solvent of DMSO/DI water (volume ratio v/v, from $3 / 1$ to $0 / 1$ ) for $72 \mathrm{~h}$. The product was finally obtained as a brick-red powder by lyophilization. The TA-COF-P was prepared without TPZ and Ce6. The TA-COF-P@C was prepared with Ce6 and without TPZ. The TBCOF-P@CT was prepared by TB-COF, loading Ce6 and TPZ. All the above samples were prepared through a similar procedure.

\section{Characterization of TA-COF and TA-COF-P@CT}

TA-COF and TA-COF-P were characterized by FT-IR to prove the occurrence of chemical reactions. XRD and BET surface were used to study the structure of TA COF. The simulated XRD pattern of TA-COF was produced from the CIF data, provided by Rahul Banerjee et al. ${ }^{2}$ using the software programs of Diamond and OriginPro. The absorption spectra of TA-COF-P@CT were measured on a spectrophotometer. The fluorescence spectra of TA-COF-P@CT were measured for TPZ with the excitation wavelength at $495 \mathrm{~nm}$ and the emission wavelength ranging from 520 to $660 \mathrm{~nm}$. For the time-dependent stability studies, the TA-COF-P@CT were incubated with saline, 
PBS, DMEM medium and RPMI 1640 medium for different time, and the change of size was measured by DLS. For the pH-dependent stability studies, the TA-COF-P@CT were incubated with different $\mathrm{pH}$ aqueous solution, and the change of size was measured by DLS.

\section{Singlet oxygen detection}

1,3-Diphenylisobenzofuran (DPBF) in ethanol $(6 \mu \mathrm{L}, 5 \mathrm{mM})$ was added to a PBS solution of free Ce6, TA-COF-P@C, TB-COF-P@CT and TA-COF-P@CT (3 mL, 5 $\mu \mathrm{M}$ Ce6). The system was then irradiated with $650 \mathrm{~nm}$ light within $60 \mathrm{~s}\left(50 \mathrm{~mW} \mathrm{~cm}{ }^{-2}\right)$. The absorption spectra of the mixture after the designated time intervals of NIR-laser irradiation were obtained on a UV-Vis spectrophotometer.

\section{Reduction of TB-COF-P@CT and TA-COF-P@CT}

The reduction of TB-COF-P@CT and TA-COF-P@CT was performed in PBS (10 mM, $\mathrm{pH} 7.4)$ at $37^{\circ} \mathrm{C}$ for $60 \mathrm{~min}$ with the sodium dithionite $(2 \mathrm{mM})$, as a chemical mimic of azo reductase. Then the TEM images of TB-COF-P@CT and TA-COF-P@CT before and after reduction was photographed. Also, the hydrodynamic size of TB-COF-P@CT and TA-COF-P@CT before and after reduction was measured by DLS. As well, The TPZ fluorescence of TB-COF-P@CT and TA-COF-P@CT before and after reduction were measured.

\section{Cell uptake}

4T1 cells were cultured by RPMI 1640 medium containing 10\% FBS, $100 \mathrm{U} \mathrm{mL}^{-1}$ penicillin, and $100 \mu \mathrm{g} \mathrm{mL}-1$ streptomycin at $37{ }^{\circ} \mathrm{C}$ in a humidified incubator containing 5\% $\mathrm{CO}_{2}$. To investigate the cellular uptake of TA-COF-P@CT, 4T1 cells were seeded 
in 6-well plate with the cell density of $2.5 \times 10^{5}$ cells per well and cultured for $24 \mathrm{~h}$ at $37^{\circ} \mathrm{C}$. Thereafter, the samples were added to the dishes separately and further incubated for $4 \mathrm{~h}$. Afterward, the cells were washed with PBS for three times and then fixed with 4\% paraformaldehyde for $15 \mathrm{~min}$ at room temperature. Hoechst 33362 was used to stain the nucleus of cells. Finally, the cells were washed with PBS and imaged by confocal microscope with $485 \mathrm{~nm}$ laser excitation for TPZ and $633 \mathrm{~nm}$ excitation for Ce6. The pin-hole diameter was set as $1 \mathrm{AU}$.

\section{ROS and hypoxia imaging}

DCFH-DA was used as the ROS fluorescence indicator to evaluate the ROS generation in cells. After seeding of the 4T1 cells on the confocal dish, the corresponding samples were added and incubated for $4 \mathrm{~h}$. Then, the cells were washed three times with PBS and incubated with DCFH-DA solution $(40 \mu \mathrm{M})$ for $30 \mathrm{~min}$. After the cells were washed with PBS three times, they were exposed to $650 \mathrm{~nm}$ laser irradiation with the power intensity of $50 \mathrm{~mW} \mathrm{~cm} \mathrm{~cm}^{-2}$ for $3 \mathrm{~min}$. Subsequently, the fluorescence images were observed on a fluorescence microscope with $488 \mathrm{~nm}$ excitation.

Image-i $\mathrm{T}^{\mathrm{TM}}$ Hypoxia Reagent, a live-cell permeable compound which increase fluorescence in environments with low oxygen concentrations, was used as fluorescence indicator to evaluate the oxygen concentration in vitro. After seeding of the $4 \mathrm{~T} 1$ cells on the confocal dish, the corresponding samples were added and incubated for $4 \mathrm{~h}$. Then, the cells were washed three times with PBS and incubated with ImageiT ${ }^{\mathrm{TM}}$ Hypoxia Reagent stock solution $(10 \mu \mathrm{M})$ for $30 \mathrm{~min}$. After the cells were washed with PBS three times, they were exposed to $650 \mathrm{~nm}$ laser irradiation with the power 
intensity of $50 \mathrm{~mW} \mathrm{~cm}{ }^{-2}$ for $3 \mathrm{~min}$. Subsequently, the fluorescence images were observed on a fluorescence microscope with $488 \mathrm{~nm}$ excitation.

\section{The expression of azo reductase of $4 \mathrm{~T} 1$ cells under different conditions}

To compare the expression of azo reductase of 4T1 cells under different conditions, 4T1 cells were seeded in the 6 -well plates at the density of $10^{8}$ cells per well. The cells were divided three groups and treated differently. The first group was incubated under standard condition (containing humidified $5 \% \mathrm{CO}_{2} / 95 \%$ air at $37{ }^{\circ} \mathrm{C}$ ) for $12 \mathrm{~h}$. The second group was incubated under hypoxic condition $\left(1 \% \mathrm{O}_{2}\right)$ for $12 \mathrm{~h}$. The third group was first incubated under standard condition for $8 \mathrm{~h}$, and then a certain amount of oil (nontoxicity, Enzo Life Sciences) was added on the upper surface of the medium to isolate the air followed by laser irradiation $\left(650 \mathrm{~nm}, 50 \mathrm{~mW} \mathrm{~cm}{ }^{-2}\right)$ for $3 \mathrm{~min}$ and further incubation for $4 \mathrm{~h}$. After the addition of cell lysis buffer to each group, the mixture was centrifuged at $5000 \mathrm{rpm}$ for $5 \mathrm{~min}$ and the supernatant was collected. Then $1 \mu \mathrm{mol}$ of NADPH, $8 \mathrm{nmol}$ of orange II was added each group separately. The concentration of azo reductase was spectrophotometrically assayed at room temperature at $513 \mathrm{~nm}$.

\section{Light-induced release in vitro}

To investigate the intracellular drug release of TA-COF-P@CT under hypoxia or normoxia condition, the 4T1 cells were first incubated under standard condition (containing humidified $5 \% \mathrm{CO}_{2} / 95 \%$ air at $37^{\circ} \mathrm{C}$ ) for $12 \mathrm{~h}$, afterward the culture plates were transferred into hypoxia $\left(1 \% \mathrm{O}_{2}\right)$ or normoxia $\left(5 \% \mathrm{CO}_{2} / 95 \%\right.$ air $)$ condition for 12 h. The hypoxia condition was made by Anaero Pack-Anaero (Mitsubishi Gas Chemical Co.Inc., Japan). Thereafter, the samples were added and further incubated for $4 \mathrm{~h}$ under 
hypoxia or normoxia condition. The following imaging process was the same as above. The laser-induced local hypoxic environment and drug release were performed as follows: the cells were incubated under standard condition and then incubated with TACOF-P@CT for 2 h, afterward a certain amount of oil (nontoxicity, Enzo Life Sciences) was added on the upper surface of the medium. After laser irradiation $(650 \mathrm{~nm}, 50 \mathrm{~mW}$ $\mathrm{cm}^{-2}$ ) for $3 \mathrm{~min}$, the cells were further cultured for $2 \mathrm{~h}$ and then imaged by fluorescence microscope.

\section{Apoptosis assay}

Apoptosis of 4T1 cells was detected using the standard 3-(4,5-dimethylthiazol-2-yl)2,5-diphenyltetrazolium bromide (MTT) assay. The cells were seeded in the 96-well plates at the density of 5000 cells per well. After $24 \mathrm{~h}$ of incubation, the cells were cultured for $12 \mathrm{~h}$ in 24 groups as follows: PBS, TPZ, TA-COF-P, TA-COF-P@C, TBCOF-P@CT, TA-COF-P@CT under different conditions (normoxia, normoxia+light, hypoxia, hypoxia+light $)\left(650 \mathrm{~nm}, 50 \mathrm{~mW} \mathrm{~cm} \mathrm{~m}^{-2}\right.$ for $\left.5 \mathrm{~min}\right)$. The procedures were treated in accordance with the manufacturer' s protocol.

\section{Animal model}

4T1 mice were bought from the Animal center of Drum-tower Hospital and performed in accord with guidelines provided by the Animal Care Committee at Drum-tower Hospital (Nanjing, China). $100 \mu \mathrm{L}$ of $4 \mathrm{~T} 1$ cells $\left(5 \times 10^{6}\right)$ in PBS suspension was injected into the right posterior side of each mouse to establish 4T1 tumors. The in vivo experiments were performed at approximately $40 \mathrm{~mm}^{3}$ of the tumor size.

\section{In vivo combined therapy}


Seven groups of 4T1 xenograft-tumor-bearing BABL/c mice were randomly divided into 7 groups (5 mice per group): (1) saline, (2) TA-COF-P with laser irradiation, (3) TPZ with laser irradiation, (4) TA-COF-P@C with laser irradiation, (5) TA-COFP@CT without laser irradiation, (6) TB-COF-P@CT with laser irradiation and (7) TACOF-P@CT with laser irradiation. All samples were administrated via tail vein, respectively. The dose of TPZ was $9 \mathrm{mg} \mathrm{kg}^{-1}$. After $12 \mathrm{~h}$, the mice except group of five were irradiated by a laser $\left(650 \mathrm{~nm}, 150 \mathrm{~mW} \mathrm{~cm}^{-2}\right)$ for $15 \mathrm{~min}$. The tumor volume and body weight of mice were measured on alternate day. The volume of tumor was calculated as follows:

$$
\text { Volume }=\frac{1}{2} a b^{2}
$$

In the equation, a represents the maximum diameter of tumor while $b$ represents the minimum diameter of tumor. Hematoxylin and eosin (H\&E) staining was carried for tumors and main organs harvested from each group at day 14.

\section{Statistical analysis}

All data are expressed as means \pm SD. Statistical differences were performed using student's t test and one-way ANOVA with OriginPro software. $\mathrm{P}<0.05$ was considered statistically significant. Vessel density and vessel number was analyzed by ImageJ. 


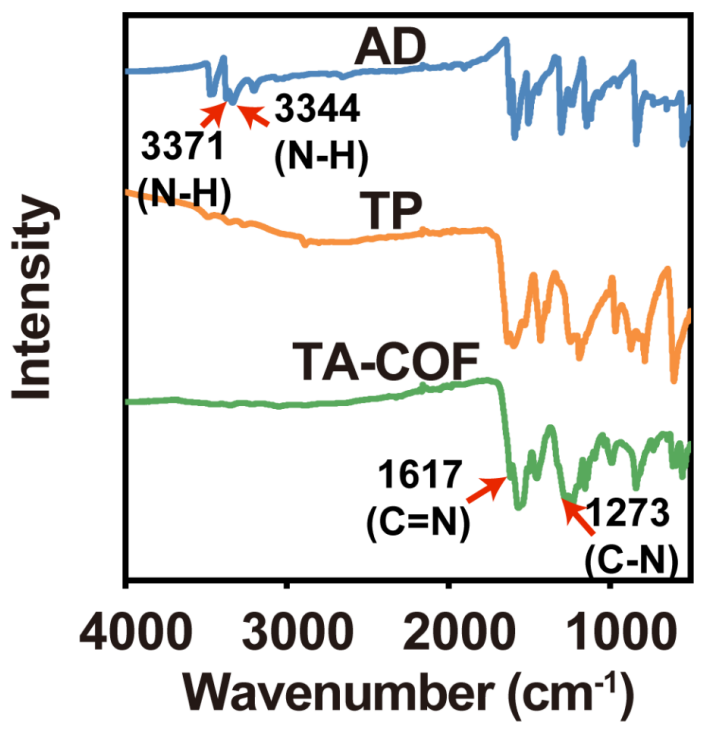

Figure S1. FT- IR spectra of AD, TP and TA-COF. 

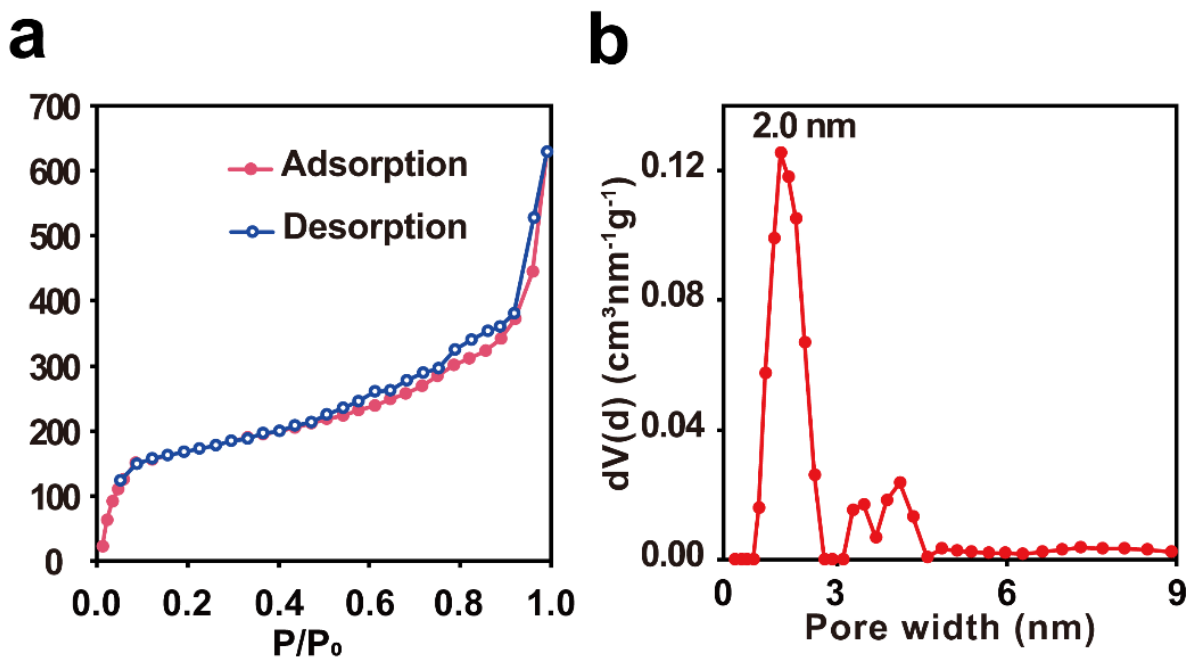

Figure S2. (a) BET surface area measurement of the TA-COF; (b) Pore size distribution of the TA-COF. 


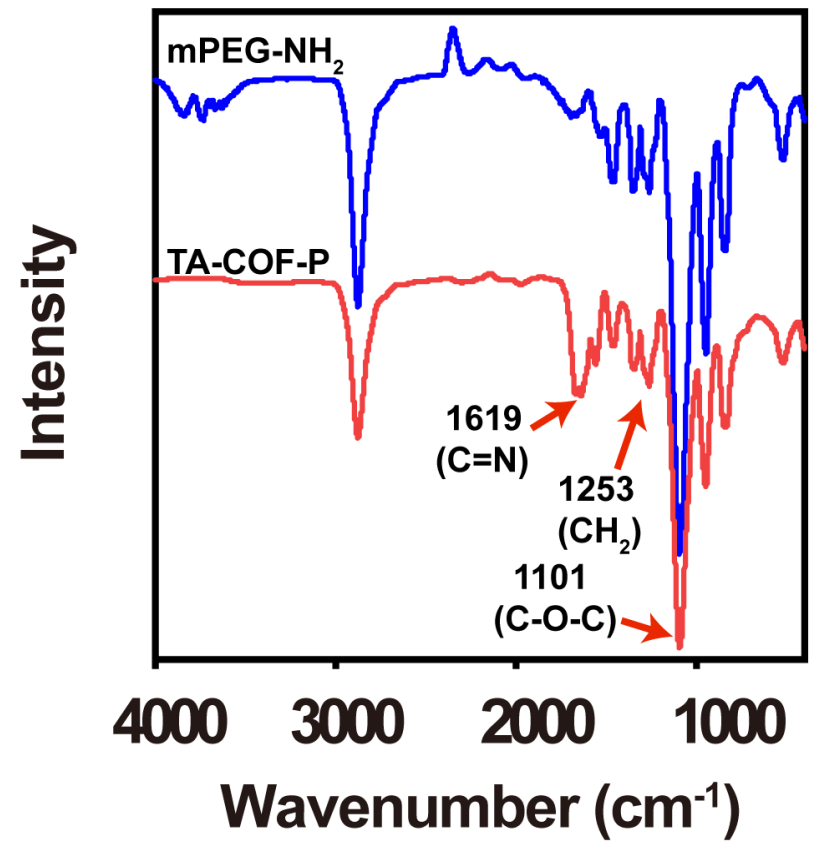

Figure S3. FT- IR spectra of mPEG-NH 2 and TA-COF-P, the new peaks of TA-COF-P at $1253 \mathrm{~cm}^{-1}\left(\mathrm{CH}_{2}\right)$ and $1101 \mathrm{~cm}^{-1}(\mathrm{C}-\mathrm{O}-\mathrm{C})$ proved that the reaction of $\mathrm{mPEG}-\mathrm{NH}_{2}$ with TA-COF. 

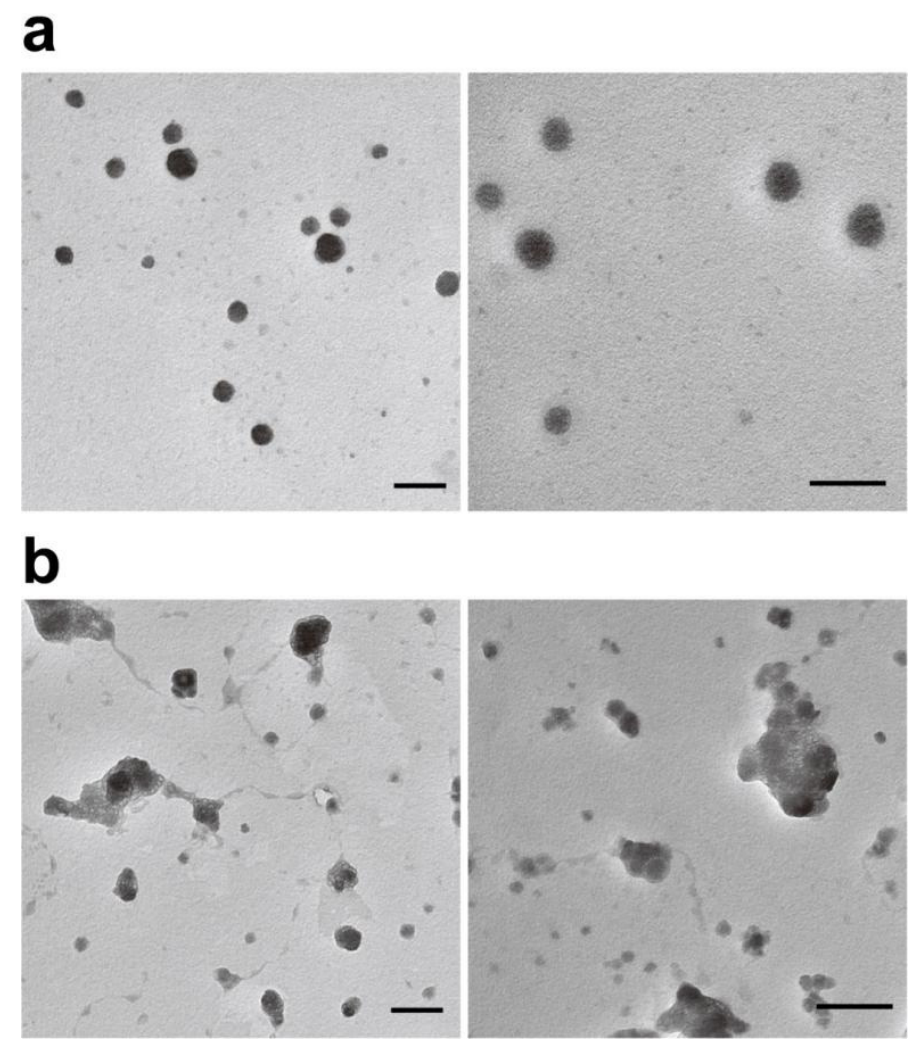

Figure S4. Extra TEM images of TA-COF-P@CT without $\mathrm{Na}_{2} \mathrm{~S}_{2} \mathrm{O}_{4}$ (a) and with $\mathrm{Na}_{2} \mathrm{~S}_{2} \mathrm{O}_{4}$ (b). Scale bar is $200 \mathrm{~nm}$. 

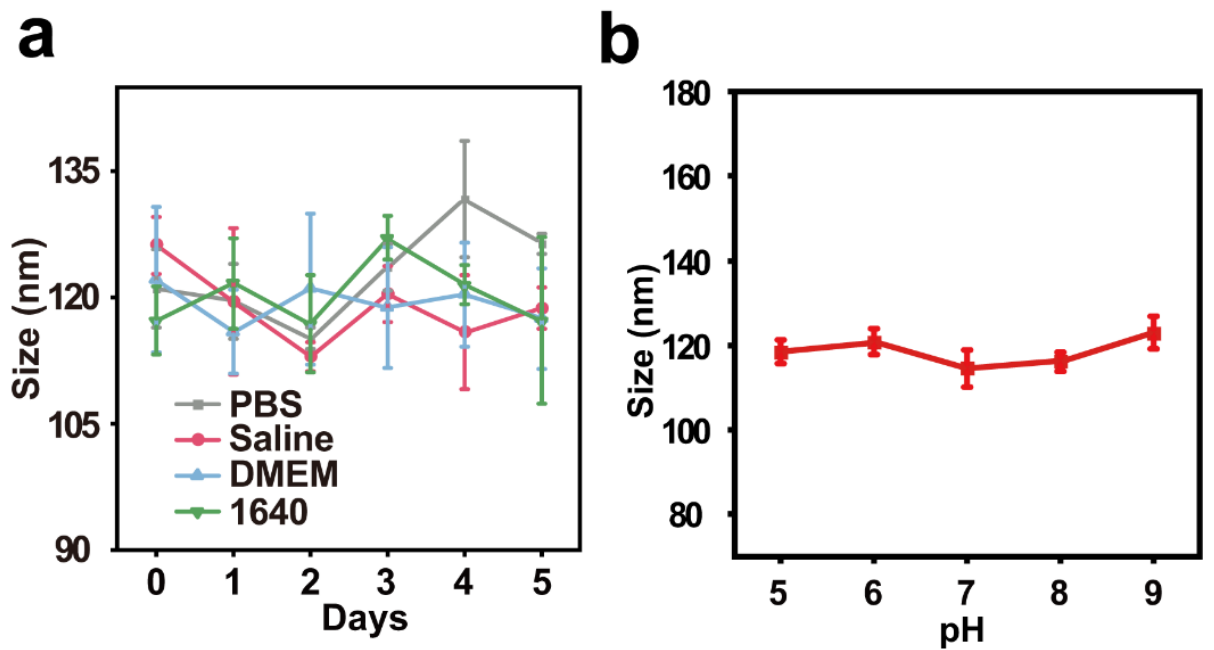

Figure S5. (a) Size of the TA-COF-P@CT in different solutions upon 5 days; (b) Size of the TA-COF-P@CT in PBS of different pH. Error bars indicated SD $(n=5)$. 

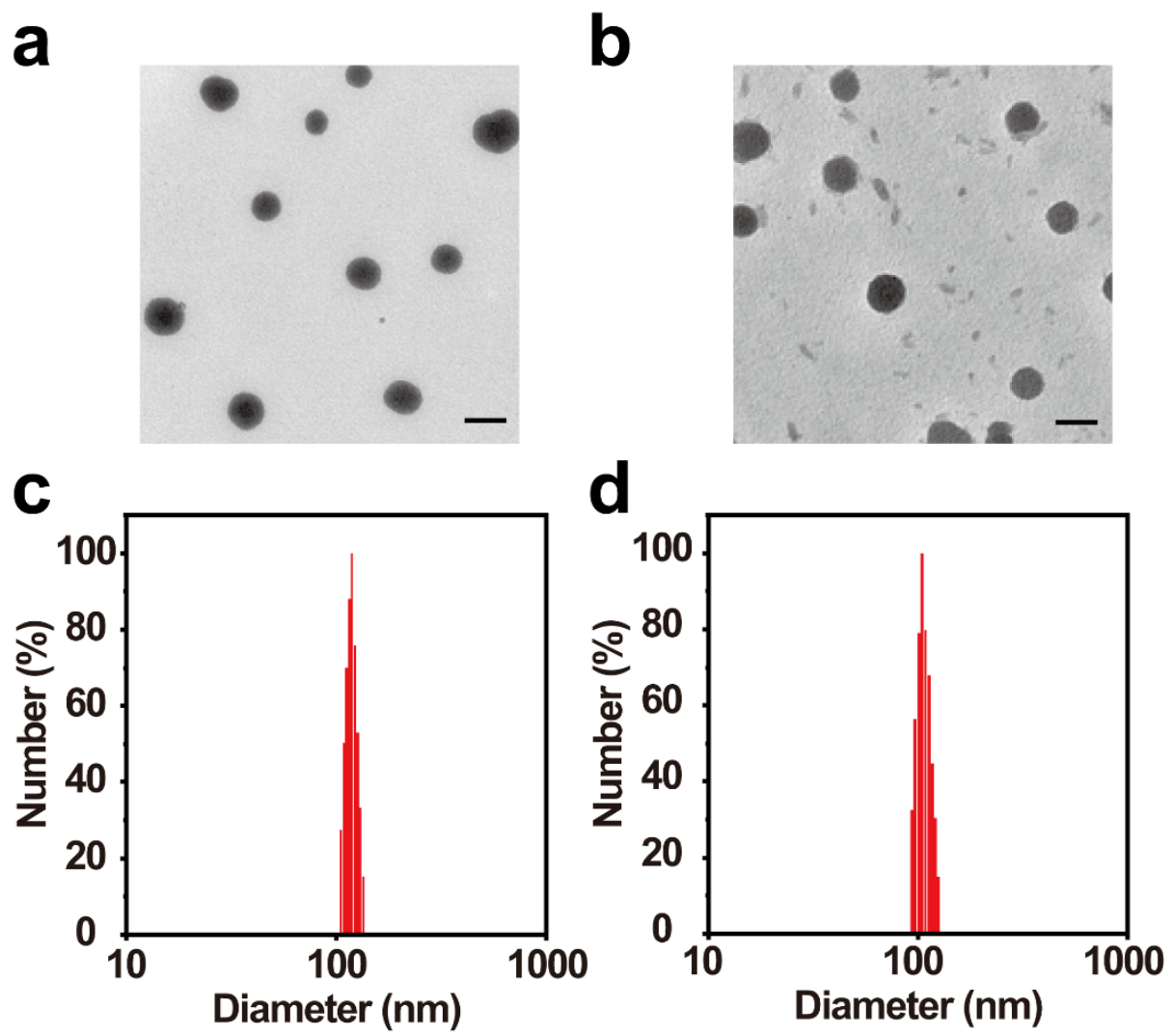

Figure S6. (a) TEM image of TB-COF-P@CT. Scale bar is 100 nm; (b) TEM image of TB-COF-P@CT after the treatment of $\mathrm{Na}_{2} \mathrm{~S}_{2} \mathrm{O}_{4}$. Scale bar is $100 \mathrm{~nm}$; (c) Hydrodynamic size distribution of TB-COF-P@CT; (d) Hydrodynamic size distribution of TB-COFP@CT after the treatment of $\mathrm{Na}_{2} \mathrm{~S}_{2} \mathrm{O}_{4}$. 


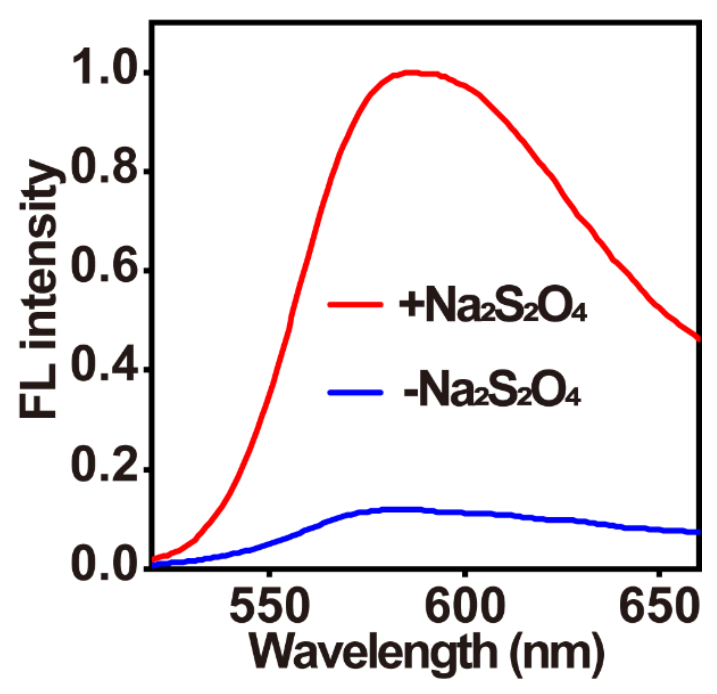

Figure S7. Fluorescence emission spectrum of TA-COF-P@CT with and without $\mathrm{Na}_{2} \mathrm{~S}_{2} \mathrm{O}_{4}, \mathrm{E}_{\mathrm{x}}=495 \mathrm{~nm}, \mathrm{E}_{\mathrm{m}}=580 \mathrm{~nm}($ for TPZ). 


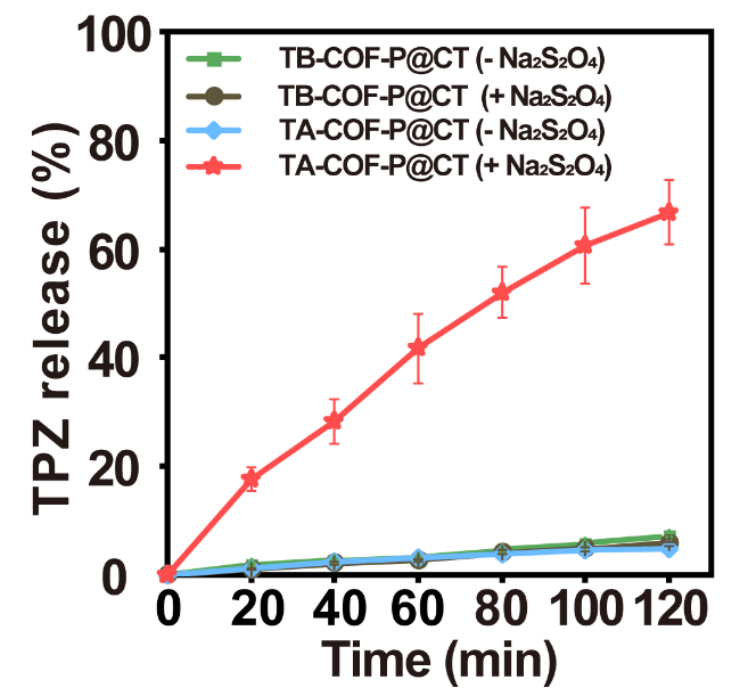

Figure S8. The release of TPZ from TB-COF-P@CT and TA-COF-P@CT with and without $\mathrm{Na}_{2} \mathrm{~S}_{2} \mathrm{O}_{4}$. Error bars indicated $\mathrm{SD}(\mathrm{n}=3)$. 


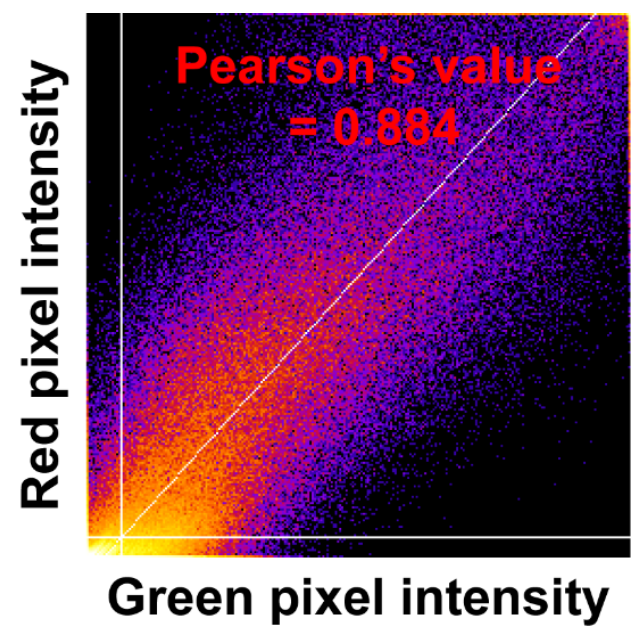

Figure S9. The representative scatter plot of colocation analysis for Ce6 (green) and TPZ (red) in cells after the incubation with TA-COF-P@CT. 


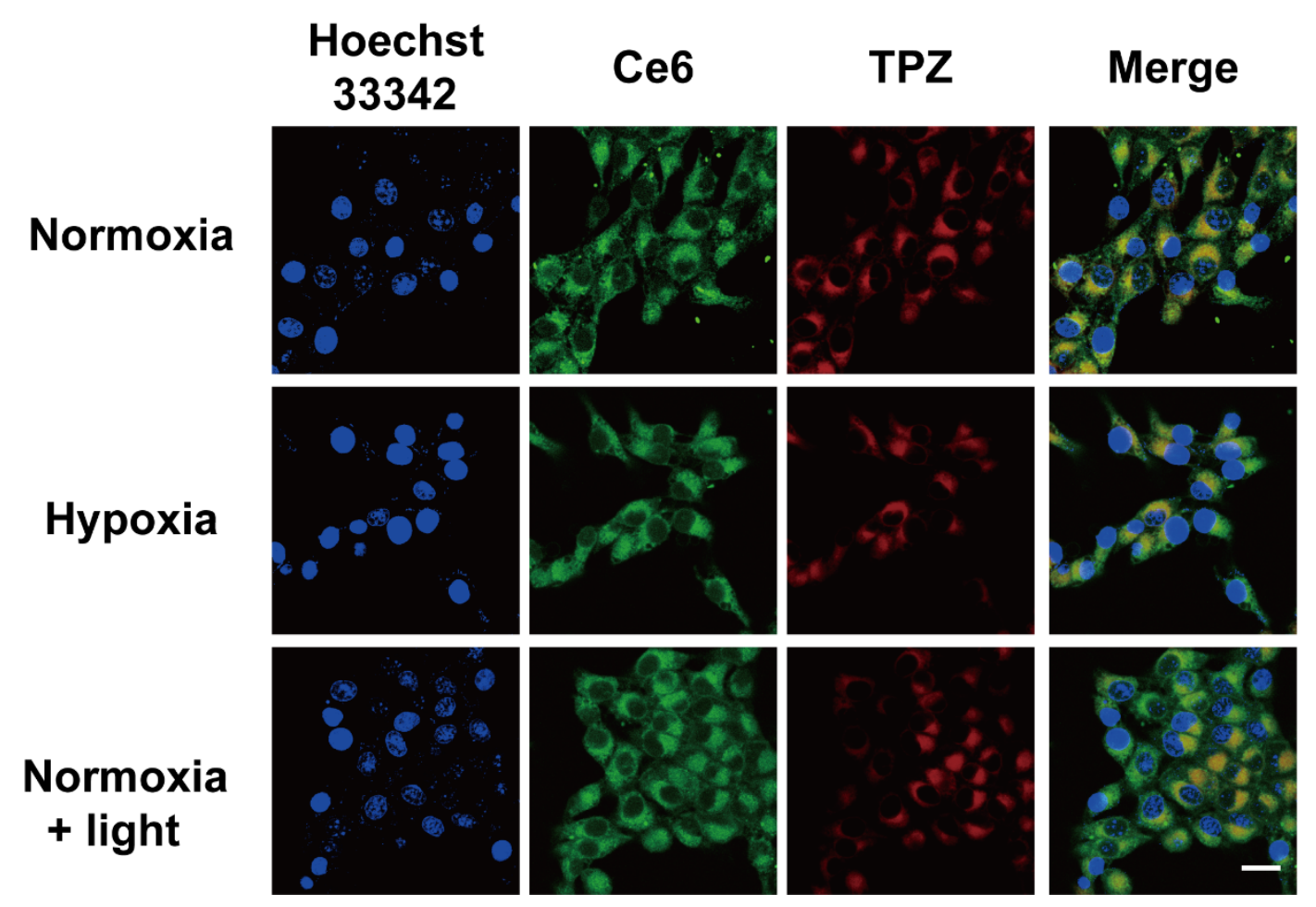

Figure S10. Confocal images of 4T1 cells incubated for TB-COF-P@CT under different conditions. Cell nuclei were labelled by Hoechst 33342. Scale bar is $20 \mu \mathrm{m}$. 
a

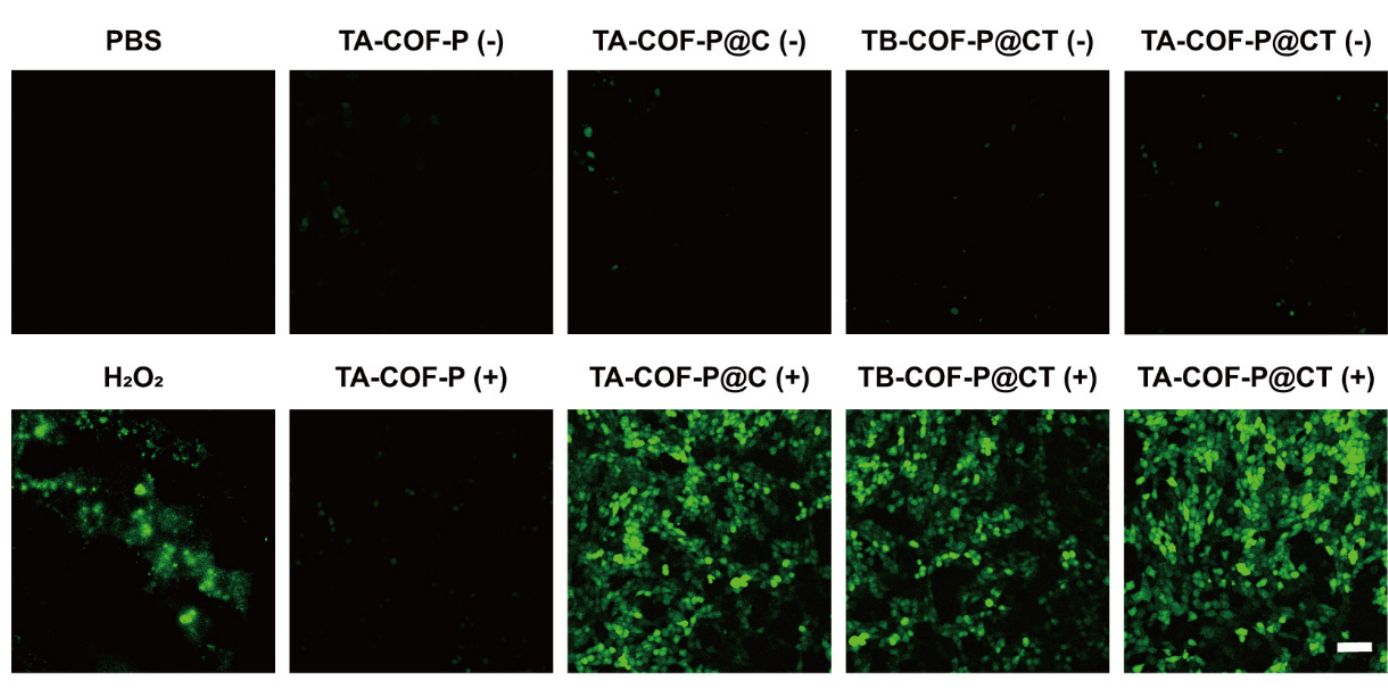

b

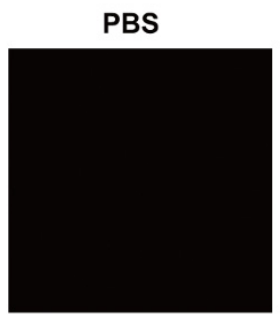

TA-COF-P (-)

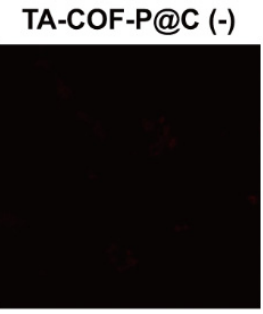

TB-COF-P@CT (-)

TA-COF-P@CT (-)
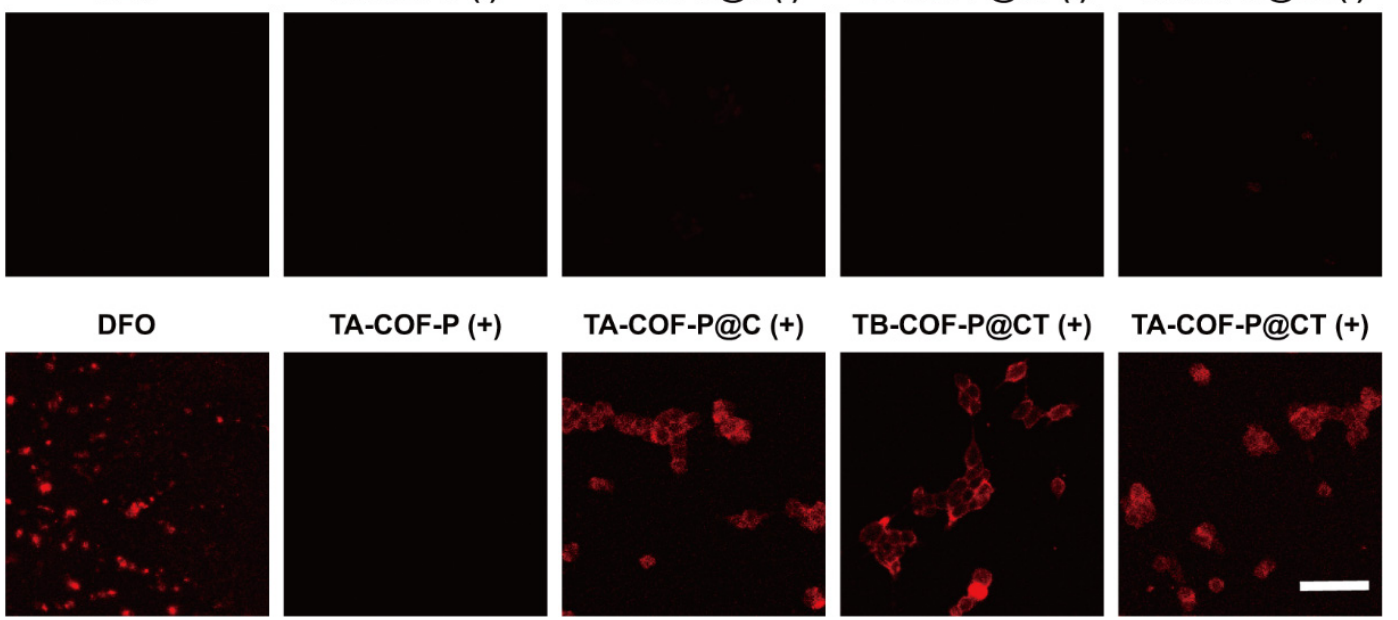

TA-COF-P (+)

TA-COF-P@C (+)

TB-COF-P@CT (+)

TA-COF-P@CT (+)
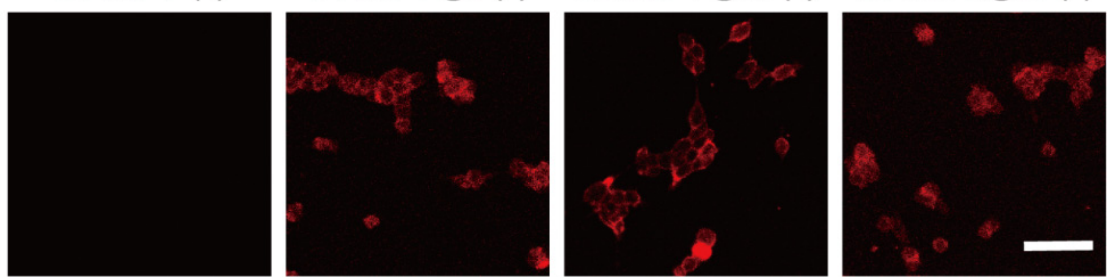

Figure S11. The intracellular ROS (a) and hypoxia (b) detection of 4T1 cells incubated with different samples and with (+) or without (-) light. Scale bar is $100 \mu \mathrm{m}$. 


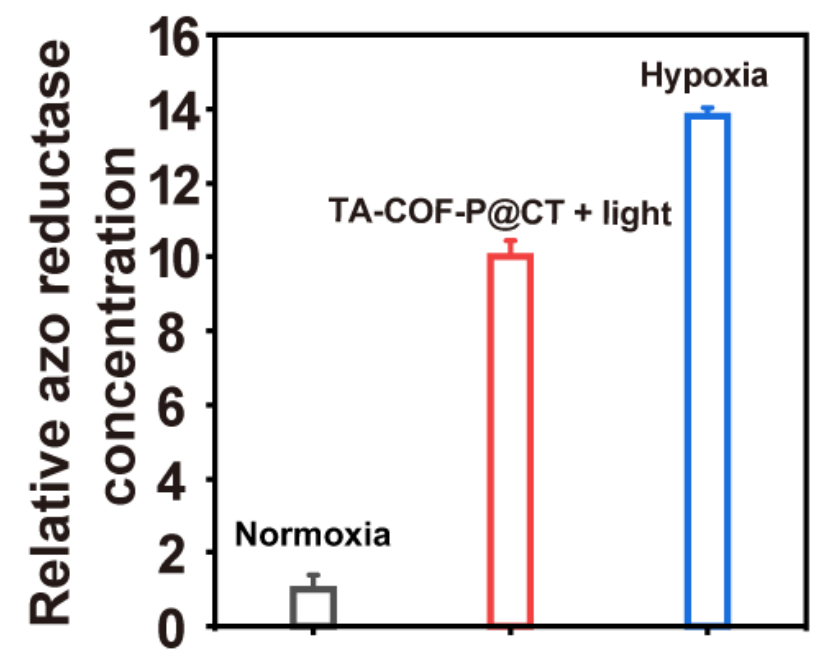

Figure S12. Relative azo reductase concentration of different groups at irradiation time of 300 s. Error bars indicated SD $(n=3)$. 


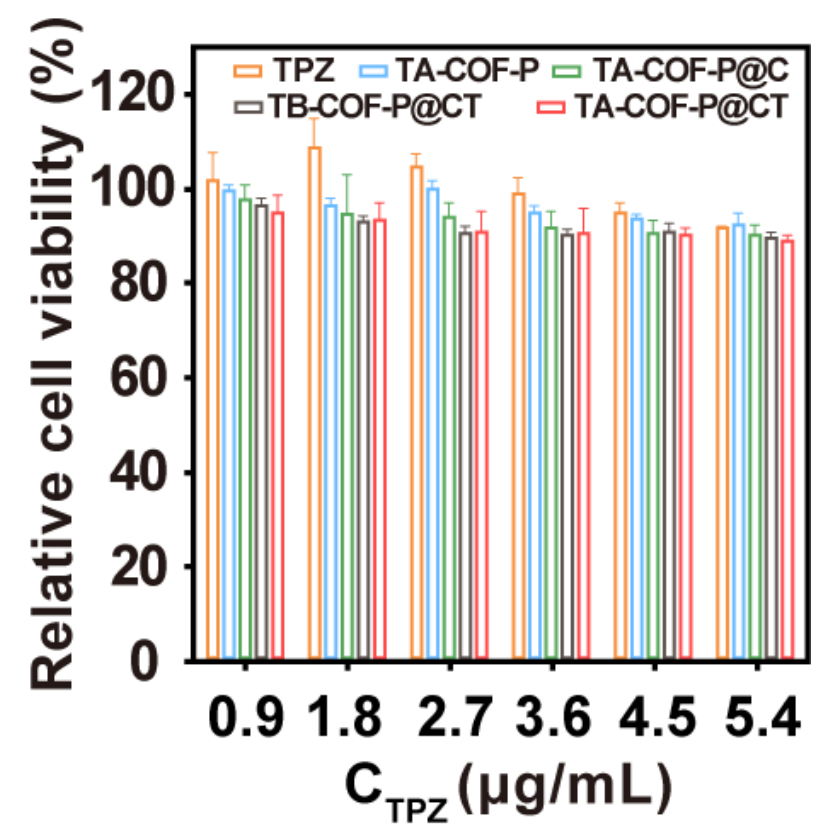

Figure S13. Relative cell viability of $4 \mathrm{~T} 1$ cells incubated for different samples under normoxia conditons. Error bars indicated SD $(n=3)$. 


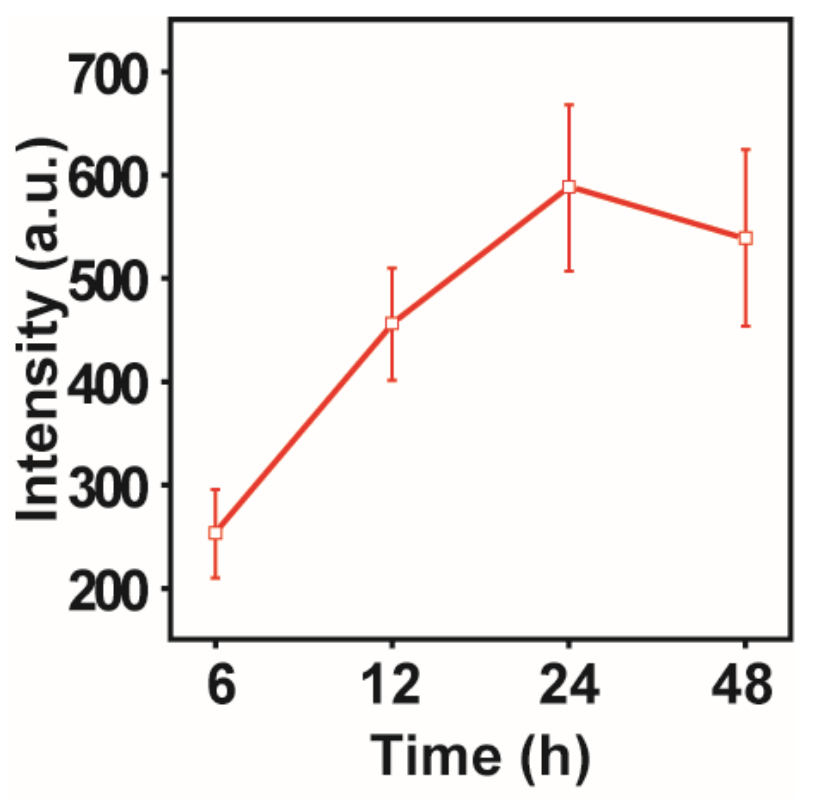

Figure S14. The normalized fluorescence intensity of tumor site at different time point at Figure 5a. Error bars indicated SD ( $\mathrm{n}=3)$. 
a

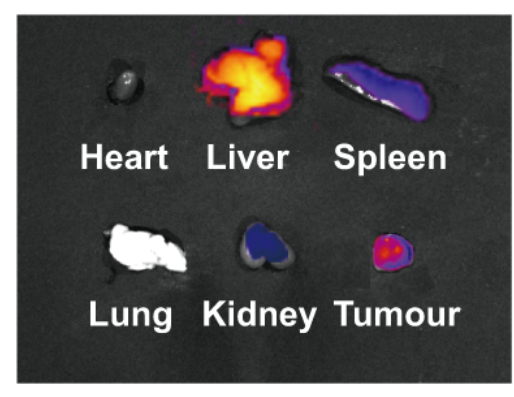

b

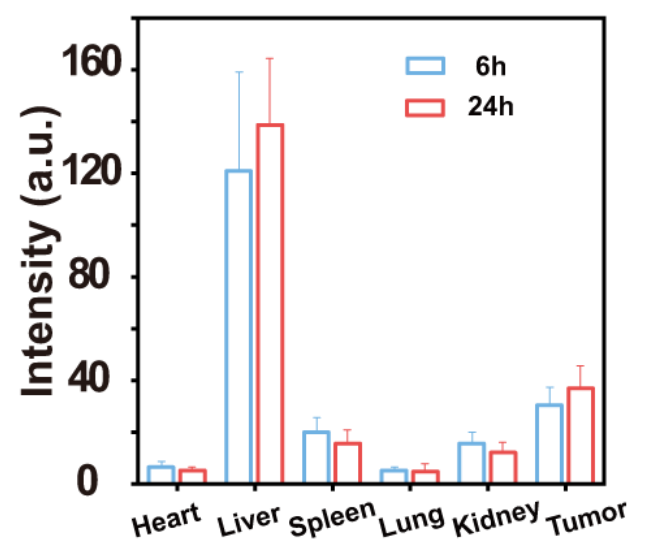

Figure S15. (a) The major organs of tumour-bearing mice were isolated and immediately imaged at $6 \mathrm{~h}$ after i.v. injection of TA-COF-P@CT; (b) The normalized fluorescence intensity of different organs at Figure 5b and S15a. Error bars indicated $\operatorname{SD}(n=3)$. 


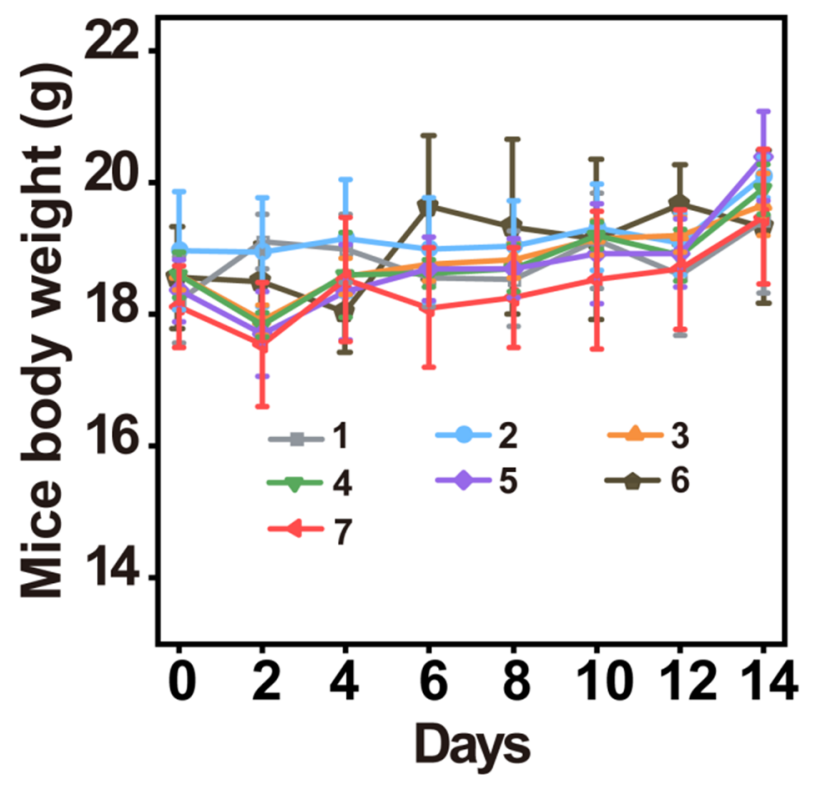

Figure S16. Body weight of mice after different treatments: (1) Saline, (2) TA-COF-P (+), (3) TPZ (+), (4) TA-COF-P@C (+), (5) TA-COF-P@CT (-), (6) TB-COF-P@CT $(+)$ and $(7)$ TA-COF-P@CT $(+)$. All data were presented as mean \pm SD $(n=5)$. 

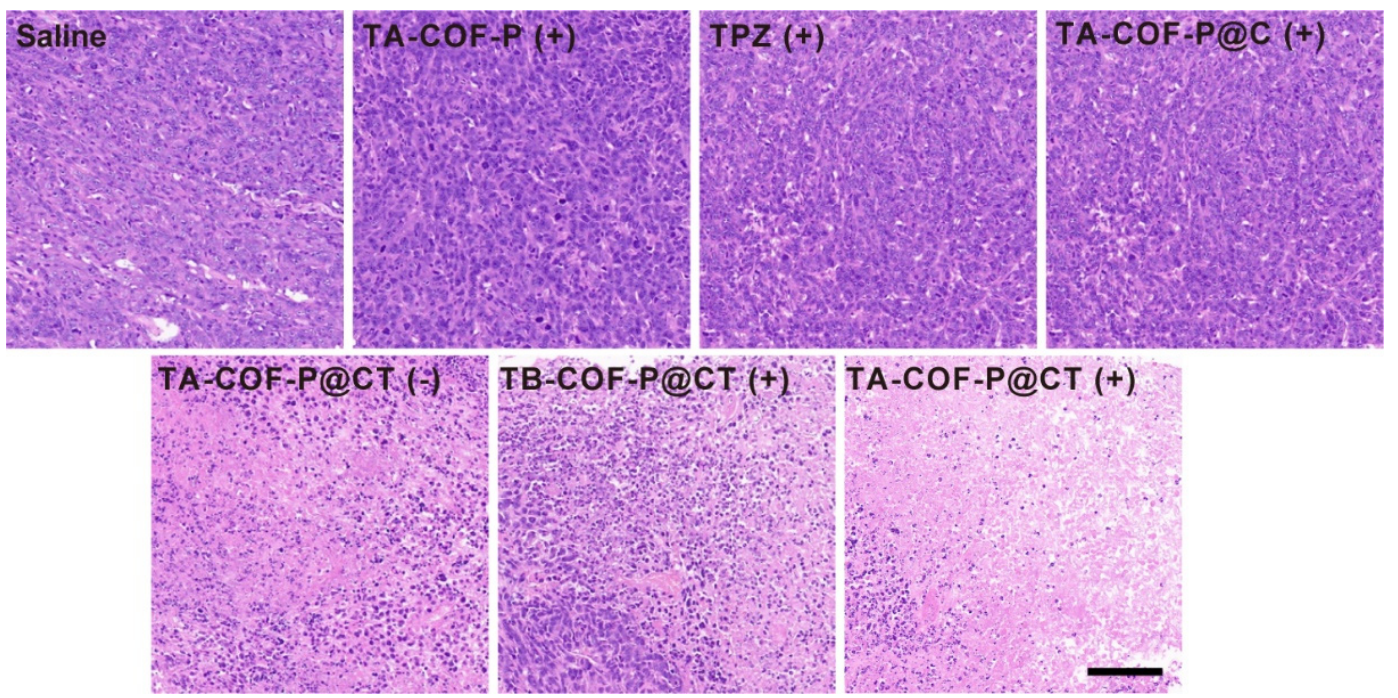

Figure S17. Histological observation of the tumor tissues after treatments. The tumor sections were stained with hematoxylin and eosin (H\&E). Scale bar is $100 \mu \mathrm{m}$. 


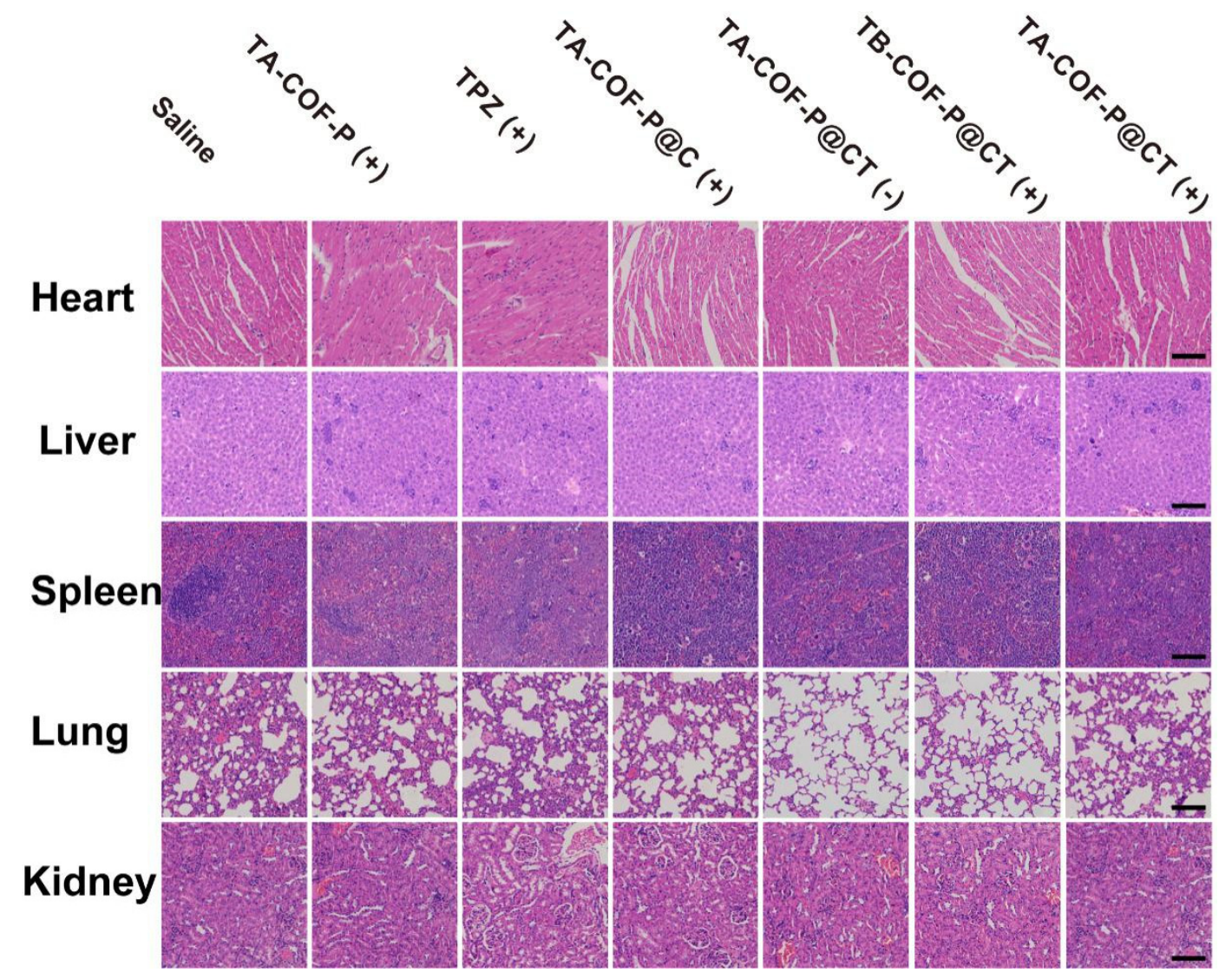

Figure S18. Micrographs of H\&E stained main organ slices from mice of different groups collected 14 days after treatments. Scale bar is $100 \mu \mathrm{m}$. 


\section{References}

(1) Sasmal, H. S.; Halder, A.; Kunjattu, H. S.; Dey, K.; Nadol, A.; Ajithkumar, T. G.; Ravindra Bedadur, P.; Banerjee, R., Covalent Self-Assembly in Two Dimensions: Connecting Covalent Organic Framework Nanospheres into Crystalline and Porous Thin Films. J Am Chem Soc 2019, 141 (51), 20371-20379.

(2) Chandra, S.; Kundu, T.; Kandambeth, S.; Babarao, R.; Marathe, Y.; Kunjir, S. M.; Banerjee, R., Phosphoric acid loaded azo (-N horizontal lineN-) based covalent organic framework for proton conduction. J Am Chem Soc 2014, 136 (18), 65703. 